\title{
IMPACTO DEL OCIO FORMATIVO MUSICAL EN LOS NIVELES DE ESCUCHA. RESULTADOS DE UNA INTERVENCIÓN CON PERSONAS MAYORES
}

\author{
IMPACT OF MUSICAL TRAINING AS A LEISURE ACTIVITY ON LISTENING \\ LEVELS. RESULTS OF A PROGRAMME WITH SENIOR CITIZENS
}

\section{IMPACTO DO TEMPO DE TREINO MUSICAL AO NÍVEL AUDITIVO. RESULTADOS DE UMA EXPERIÊNCIA COM PESSOAS MAIS VELHAS}

\author{
Macarena CUENCA-AMIGO* \& Mercedes ALBAINA HERNÁNDEZ** \\ * Universidad de Deusto, ** Musikene
}

\begin{tabular}{|c|c|}
\hline & $\begin{array}{r}\text { Fecha de recepción: } 01.111 .2018 \\
\text { Fecha de revisión: 19.III.2018 } \\
\text { Fecha de aceptación final: 16.IV.2018 }\end{array}$ \\
\hline $\begin{array}{l}\text { PALABRAS CLAVE: } \\
\text { ocio } \\
\text { educación } \\
\text { música } \\
\text { niveles de escucha }\end{array}$ & $\begin{array}{l}\text { RESUMEN: El objetivo de este estudio consistió en analizar si la intervención educativa a } \\
\text { través de un programa de Ocio Formativo Musical tenía un impacto significativo en los niveles } \\
\text { de escucha sensorial, afectivo y analítico en personas mayores con y sin estudios musicales } \\
\text { previos. Para ello, se diseñó una escala de medición propia y se aplicó a una muestra de } \\
37 \text { estudiantes mayores de } 50 \text { años de la Universidad de Deusto (Bilbao, España). Tanto la } \\
\text { escala en su conjunto, como cada una de las subescalas de los distintos niveles de escucha } \\
\text { mostraron una adecuada fiabilidad, con valores de alfa de Cronbach superiores a o,8. El cues- } \\
\text { tionario fue administrado en el aula el primer y el último día de clase tras la audición de un } \\
\text { mismo fragmento musical. Las mediciones anteriores y posteriores a la intervención educati- } \\
\text { va fueron comparadas aplicando el estadístico de la t-student para muestras relacionadas. La } \\
\text { investigación concluyó que el único nivel de escucha afectado de manera significativa tras la } \\
\text { intervención de Ocio Formativo Musical estudiada fue el analítico y, afinando más, el impacto } \\
\text { solo fue destacable para los individuos de la muestra sin estudios musicales previos. }\end{array}$ \\
\hline
\end{tabular}

CONTACTO CON LOS AUTORES: MACARENA CUENCA-AMIGO. Universidad de Deusto. Deusto Business School. Hermanos Aguirre 2, 48014 Bilbao, España. Tel. 94413900o. E-mail. macarena.cuenca@deusto.es 


\begin{tabular}{|c|c|}
\hline $\begin{array}{l}\text { KEY WORDS: } \\
\text { leisure } \\
\text { education } \\
\text { music } \\
\text { listening levels }\end{array}$ & $\begin{array}{l}\text { ABSTRACT: The goal of this study consisted of analysing whether educational intervention } \\
\text { by means of a Musical Training as a Leisure Activity programme had any significant impact on } \\
\text { sensorial, affective and analytical listening levels among senior citizens who had and had not } \\
\text { previously studied music. To do so, we designed our own measurement scale and applied it } \\
\text { to a sample of } 37 \text { students over } 50 \text { years of age at the University of Deusto (Bilbao, Spain). } \\
\text { Both the scale as a whole and each of the subscales of the different listening levels presented } \\
\text { adequate reliability, with Cronbach's alpha values greater than o.8. The questionnaire was } \\
\text { administered in the classroom on the first and last day of class, after hearing the same piece of } \\
\text { music. The measurements before and after the educational intervention were compared by } \\
\text { applying a Student's t-test for paired samples. The research concluded that the only listening } \\
\text { level to have been significantly affected by the Musical Training as a Leisure Activity pro- } \\
\text { gramme was the analytical level, and when examining this in greater detail, it is noted that the } \\
\text { impact was only prominent among members of the sample who had not studied music before. }\end{array}$ \\
\hline $\begin{array}{l}\text { PALAVRAS-CHAVE: } \\
\text { lazer } \\
\text { educação } \\
\text { música } \\
\text { níveis de audição }\end{array}$ & $\begin{array}{l}\text { RESUMO: O objetivo deste estudo foi analisar se a intervenção educacional através de um } \\
\text { programa de lazer de treino musical teria um impacto significativo nos níveis de audição sen- } \\
\text { sorial, afetiva e analítica em idosos com e sem estudos musicais prévios. Para este fim, dese- } \\
\text { nhámos e aplicámos uma escala de auto-medição a uma amostra de } 37 \text { alunos com mais de } 50 \\
\text { anos de idade da Universidade de Deusto (Bilbao, Espanha). Tanto a escala total como cada } \\
\text { uma das subescalas dos diferentes níveis de audição apresentaram confiabilidade adequada, } \\
\text { com valores de alfa de Cronbach maiores que o,8. O questionário foi administrado na sala de } \\
\text { aula no primeiro e último dias de aula depois de ouvir o mesmo fragmento musical. Os efeitos } \\
\text { da intervenção educacional foram comparadas usando a estatística t-student para amostras } \\
\text { relacionadas. Os resultados permitem concluir que o único nível de audição significativamen- } \\
\text { te afetado pela intervenção de treino musical estudado foi o analítico. Além disso, o impacto } \\
\text { foi notável apenas para os indivíduos da amostra sem estudos musicais prévios. }\end{array}$ \\
\hline
\end{tabular}

\section{Introducción}

El Ocio Formativo Musical (en adelante OFM) es un constructo de triple perfil -humanista, educativo y estético-, que trata de facilitar una vivencia de ocio valioso (Cuenca, 2014) en torno a la música clásica a través de la formación. El OFM se concibe en el marco de la educación no formal ya que surge de la búsqueda por crear formas alternativas de educación musical, distintas a la propiamente académica o escolar, pretendiendo generar un aprendizaje en los educandos, a través de programas realizados con el propósito específico de favorecer la escucha musical consciente (Albaina, 2015). A pesar de que el OFM puede estar dirigido a cualquier colectivo educativo, este estudio se centra en las personas mayores de 50 años, un segmento de la población cada vez más relevante (Abellán \& Ayala, 2012) y que atraviesa un periodo de su vida en el que el ocio comienza a cobrar una importancia especial y puede contribuir al proceso de envejecimiento activo y satisfactorio (Cuenca-Amigo \& San Salvador del Valle, 2016)

Uno de los grandes misterios y de las grandes riquezas del lenguaje de la música procede del hecho de que una misma composición puede suscitar en los oyentes reacciones diversas e, incluso, contrapuestas. Para Small (2006:38), "la vivencia que el oyente tiene de la música es esencialmente privada". Esta variabilidad es posible, incluso, en el mismo oyente en distintos contextos o momentos de su proceso vital. La naturaleza subjetiva de la respuesta al estímulo musical, lleva a muchos autores a considerar que la significación de la música hay que buscarla en el oído de los que escuchan antes que en la música misma (Cámara, Cañada, Albaina \& Larrinaga, 2012; MacDonald, Hargreaves \& Miell, 2012; Sloboda, 2012; Szendy, 2015). En esta línea, las teorías de Horowitz (2012) explican tanto la manera en que el sonido nos afecta, como la capacidad que tenemos de aprender a manipularlo, llegando a afirmar que somos lo que escuchamos.

Además, muchos autores tienen en cuenta la importancia de la actitud de las personas ante el hecho musical (Delalande, 2013; Schafer, 2013). Swanwick (2006) relaciona la actitud de las personas hacia la música, con el poder que ésta tiene de evocar en ellas experiencias significativas. Para Bonds (2014:19-20) el papel de los oyentes es determinante en la transformación de las actitudes hacia la música y sostiene que todas las variaciones en la escucha son "el producto de un cambio mucho más amplio en las actitudes ante el propio acto de la percepción, ante el modo en el que un sujeto (el oyente) aprehende un objeto (la obra musical)".

Pero hay otros factores individuales que determinan el modo de escuchar de las personas. Algunos de ellos están directamente asociados a la música, como son la habilidad en la identificación y conexión de parámetros musicales, la musicalidad, la memoria auditiva, la educación en la escucha, el entrenamiento o los gustos y preferencias por cada estilo de música. Otros influyen de manera indirecta como, por ejemplo, la edad, los 
factores socio-culturales e incluso la personalidad (Ball, 2010; Blacking, 2006; Dahlhaus \& Eggebrecht, 2012; Drösser, 2012; Levitin, 2011; Sacks, 2015). Desde la perspectiva del OFM es importante considerar que existen estas diferencias, sobre todo a la hora de diseñar los planteamientos didácticos, con el fin de que la escucha de la música sea una experiencia de ocio satisfactoria para el mayor número de personas posible (Albaina, 2015).

Uno de los puntos de partida de la presente investigación es el reconocimiento de que el hecho musical es susceptible de ser captado por las personas en tres niveles' de escucha complementarios: el sensorial, el afectivo y el analítico (Copland, 2008; Delalande, 2013; Sloboda, 2012; Swanwick, 2006; Willems, 2001).

El nivel sensorial se corresponde con el plano fisiológico y es imprescindible para acceder a los siguientes niveles de escucha. Su estudio se aborda desde el campo de la percepción y ha sido tema de gran interés para investigadores de diversas áreas del conocimiento, como la Fisiología, la Física -en la rama de la Acústica- la Medicina, la Psicología y, cómo no, la Música. Aun partiendo de la premisa de que el ámbito de actuación del OFM es tangencial a las áreas citadas, es importante comprender los mecanismos psicofisiológicos implicados en los procesos perceptivos del hecho musical, con el fin de entender las reacciones de los oyentes y así diseñar estrategias didácticas eficaces. Muchos autores han centrado su mirada en la comprensión del funcionamiento propio de la percepción musical, con fines educativos o estéticos (Ball, 2010; Boulez, Changeux \& Manoury, 2016; Levitin, 2011; Sacks, 2015; Sloboda, 2012; Small, 2006; Szendy, 2015). Para Bonds (2014:21) es crucial porque "podemos creer o no creer lo que vemos, pero solemos ver lo que creemos. Lo mismo cabe decir sobre la escucha. En la música, percibimos lo que estamos dispuestos a percibir".

El nivel afectivo, que puede tener lugar al mismo tiempo que el nivel sensorial, se corresponde con el plano de las emociones. Willems (2001:55) afirma que "si la sensorialidad auditiva es el punto de partida, la base misma de la musicalidad, la sensibilidad afectivo-auditiva es quizá su centro". Para Swanwick (2006) tras una primera impresión de los sonidos a nivel sensorial, las personas nos vemos afectadas por la cualidad expresiva de la música vinculada a un proceso psicológico, muchas veces inconsciente, que él llama de imitación. Otros autores prefieren utilizar el término identificación, en lugar de imitación, para dar explicación al origen del impacto emocional de la música en las personas. Según Alvin (1997), la música expresa sentimientos que surgen de una situación y que no tiene por qué ser imitativa. Esta autora alude a Aristóteles cuando menciona el principio de Iso en relación al cual "lo semejante actúa sobre lo semejante" (Alvin, 1997:109). También Benenzon (2011) utiliza el principio de Iso para explicar cómo -dentro de un completo mosaico de sonoridadeshay una serie de patrones estructurados, que permiten un canal de comunicación a nivel afectivo en el proceso de escucha musical ya que para él cada ser humano tiene una identidad sonora que lo caracteriza y lo diferencia del otro. Para Blacking (2006), lo que realmente conmueve a las personas es el contenido humano de los sonidos humanamente organizados. Gabrielsson (2011) ha investigado las reacciones a la música desde una perspectiva psicológica y ha acuñado el término strong experiences with music (SEM) para distinguir aquellas experiencias a través de la escucha, que nos hacen trasladarnos de la posición de observadores auditivos neutrales -es la que ocuparíamos al poder describir el objeto sonoro de manera más o menos objetiva, basándonos en la percepción-, a la de sujetos implicados emocionalmente a través de la escucha o, lo que es lo mismo, afectados por la música.

Muchos investigadores han estudiado las respuestas afectivas a la música a partir de verbalizaciones de los escuchantes o del análisis de determinados tipos de respuesta fisiológica (Drösser, 2012; MacDonald, Hargreaves \& Miell, 2012; Sloboda, 2012) y han llegado, entre otras, a la conclusión de que tanto las expectativas del escuchante como los factores contextuales, pueden favorecer las experiencias emocionales respecto a la música. Estas se manifiestan en las personas mayores principalmente en dos sentidos. Uno de ellos hace referencia al alivio de tensiones psíquicas a partir de la inducción a estados de catarsis emocional a través de la escucha musical. El segundo considera la dimensión afectiva-emocional desde la perspectiva de la espiritualidad humana y concluye que la vivencia de ocio, tal como se contempla desde el OFM, va asociada al bienestar espiritual de la persona.

Desde los programas de OFM, y a partir de estrategias didácticas diseñadas para facilitar a los oyentes pautas de escucha, se puede favorecer el incremento de expectativas acerca de lo que sucede en la organización de un discurso musical pudiendo ser estimulado, así, el nivel afectivo.

El nivel analítico se corresponde con el plano cognitivo. En este sentido, los programas de OFM, con su perfil educativo, contemplan el hecho de que facilitar al oyente puntos de referencia le permite realizar una serie de procesos cognitivos con los que tomar conciencia de los elementos sonoros y su organización para, así, atribuirles un significado y disfrutar de la audición (Albaina, 2015). 
El significado en la música es un tema estudiado desde diversas áreas del conocimiento: la filosofía, la estética musical y la psicomusicología, entre las más relevantes (Boulez, Changeux \& Manoury, 2016; Levitin, 2011; Sloboda, 2012). El oyente puede intentar extraer un significado concreto en una audición a partir del análisis, si bien es cierto que su acercamiento a la música en este nivel de escucha puede inclinar la atención hacia los componentes de índole emocional del discurso sonoro, o hacia los que lo configuran desde la misma esencia del lenguaje musical. En cualquier caso, la ampliación del horizonte musical gracias a una comprensión más profunda de cómo actúa la música y por qué, servirá a quienes - ya en este nivelpueden considerarse plenamente escuchantes, para disfrutar la música de una manera más plena (Ball, 2010). Pero ambos tipos de análisis no son excluyentes y mucho menos incompatibles $y$, por otra parte, el mismo discurso musical tiene para cada oyente un significado concreto. Es aceptado que, a lo largo de la historia, los creadores se han servido de determinados recursos estilísticos y técnicas de composición para comunicar emociones de "formas misteriosamente bellas" (Rosen, 2012). Muchos autores se han fijado en el valor que, desde una audición activa, puede tener el hecho de que ambos análisis se complementen (Dahlhaus \& Eggebrecht, 2012; Delalande, 2013; Meyer, 2009; Sloboda, 2012; Small, 2006).

Para Swanwick (2006:99), el análisis auditivo tiene que ver con el juego imaginativo, que él define como "un concepto psicológico que tiene su correlación musical en nuestro modo de responder y de crear relaciones formales, aportando a la música una amplia serie de expectativas, especulando y previendo un futuro de progresión musical". Y esto es susceptible de aprenderse a través del OFM. Aaron Copland que, como compositor, también asumió la importancia de que los oyentes de música clásica tuvieran una formación, aún elemental, que les permitiera analizar lo escuchado para comprenderlo y les vinculara al disfrute de la música en directo, afirmaba: "Es muy importante para todos nosotros que nos hagamos más sensibles a la música en su plano puramente musical. Después de todo, es una materia verdaderamente musical lo que se está empleando" (Copland, 2008:33). Además, como señala Blacking (2006) la música no puede expresar nada extramusical a menos que la experiencia a la cual se refiere ya exista en la mente de quien escucha.

Tal y como se desprende de la literatura, los tres niveles de escucha se ven enlazados (Ball 2010; Boulez, Changeux \& Manoury, 2016; Delalande, 2013; Sacks, 2015; Sloboda, 2012; Swanwick, 2006), reforzando el disfrute de la música a través de una audición más plena y desde esta perspectiva son diseñados los programas de OFM. El objetivo de este estudio consiste en analizar si la intervención educativa a través de un programa de OFM tiene un impacto significativo en los niveles de escucha sensorial, afectivo y analítico en personas con y sin estudios musicales previos. La hipótesis es que el OFM impacta de forma positiva en los tres niveles de escucha en ambos colectivos.

\section{Metodología}

Con el objetivo de analizar el impacto del OFM en los niveles de escucha, se diseñó una metodología de carácter cuantitativo basada en una nueva escala de medición. Los siguientes apartados desarrollan la muestra seleccionada para la intervención, el proceso de construcción de la escala, así como el procedimiento de recogida y análisis de datos.

\subsection{Participantes}

La población de referencia para la realización de este estudio ha sido el conjunto de alumnos y alumnas mayores que participan en cursos de OFM en Euskadi, donde hay varias entidades y asociaciones que contribuyen al desarrollo formativo y cultural del colectivo de personas mayores. La presente investigación se centra en el ámbito universitario por considerar que, a priori, esta labor será acometida por las universidades de manera más sistemática, con mayor disposición de recursos y con un rigor académico que implique una eficacia evidente y una solidez más duradera. Durante el curso 2016-2017 encontramos planteamientos relacionados con el OFM en la programación de 24 de las 45 universidades de la Asociación Estatal de Programas Universitarios para Personas Mayores (AEPUM), datos que reflejan la implantación del OFM en algo más de la mitad $(53,33 \%)$ de los programas universitarios para personas mayores.

Para proceder a la selección de la muestra, se llevó a cabo un muestreo no probabilístico (Malhotra, 2004) y se seleccionó como estudio de caso los cursos de OFM impartidos en la Universidad de Deusto durante el segundo semestre del curso 2015-2016. En dicha institución la formación permanente dirigida a personas mayores se canaliza a través de la Escuela de Ciudadanía (Deustobide) y durante el periodo seleccionado para el estudio fueron dos los cursos de OFM ofertados. El primer curso, titulado "Vidas sonoras. Retratos y relatos de músicos a través de su obra", pertenecía al programa Ocio Cultural Universitario y contaba 
con 20 personas mayores inscritas. El segundo curso, titulado "Géneros y ciclos musicales", pertenecía al Titulado Universitario en Cultura y Solidaridad y contaba con 44 personas mayores inscritas. La muestra objetivo constaba, por tanto, de 64 estudiantes. Sin embargo, la necesidad de medición el primer y último día de clase, unida a las faltas de asistencia o faltas de puntualidad de los estudiantes, así como al requerimiento de responder a la totalidad del cuestionario, supuso una reducción de la muestra real a 37 individuos.

A continuación describimos brevemente los datos sociodemográficos de los 37 encuestados seleccionados. En relación a los tramos de edad, 16 individuos tenían entre 50 y 64 años (43\%); 19 correspondían al intervalo de entre 65 y 74 años (51\%) y tan solo 2 personas pertenecían al tramo de 75 o más años (5\%). Con respecto al género, 15 de los entrevistados eran hombres (41\%) y 22 eran mujeres (59\%). En lo referente al nivel de estudios, 6 individuos contaban únicamente con "Estudios primarios (Educación General Básica)" (16\%), 11 con "Estudios secundarios (Bachiller, FP)" (30\%) y 20 con "Estudios superiores (Diplomatura, Licenciatura, etc.)" (54\%). Finalmente, del total de la muestra, 24 no contaban con estudios musicales previos (65\%) y 13 tenían algún tipo de estudios musicales previos (35\%).

\subsection{Instrumento}

Teniendo en cuenta el marco teórico analizado y la inexistencia de escalas que se ajustaran plenamente al constructo de OFM, se optó por la creación de una escala propia, que centrara su atención en los tres niveles de escucha identificados: sensorial, afectivo y analítico. Para la elaboración de dicha escala se revisaron otras escalas relacionadas con la percepción de las artes escénicas y musicales en general (Boerner \& Jobst, 2013; Rössel, 2011) y con la percepción de la música clásica en particular (Albaina y Cámara, 2010; Blasco, 1996; Chin \& Rickard, 2012; Gabrielsson, 2002; Law \& Zentner, 2012; Thompson, 2007).

La escala completa se compone de treinta ítems, diez para cada nivel de escucha. Cada ítem contiene una afirmación para la que se debe marcar el grado de acuerdo o desacuerdo, atendiendo a una escala Likert de 7 puntos. El valor 1 significa "completamente en desacuerdo" y el 7 "completamente de acuerdo". Según González y Pazmiño (2015), la utilización de una escala Likert de 7 puntos contribuye a mejorar el nivel de fiabilidad con respecto a la escala de 5 puntos. En este caso, el nivel de fiabilidad de la escala en su conjunto, medido con el estadístico del alfa de Cronbach, arroja un valor de 0.951. A continuación, se presenta el detalle de la construcción de cada uno de los tres niveles de escucha.

En primer lugar, el nivel sensorial recoge afirmaciones relacionadas con el enfoque de la atención de los oyentes hacia una serie de estímulos sonoros que provienen de la música, al tiempo que se busca sondear sobre la toma de conciencia de dicha actitud atenta. Para acotar la indagación de esta actitud de "escuchante" frente al estado de "oyente" en los sujetos, interesa conocer sus propias reflexiones acerca de su grado de distracción cuando suena la música, si la focalización de su atención auditiva es intencionada y dirigida hacia determinados parámetros musicales previamente establecidos y ordenados en categorías y también que recapaciten sobre qué elementos sonoros destacan y si el conjunto provoca en ellos alguna sensación física. El alfa de Cronbach asciende a 0.820 para la sub-escala sensorial, que contiene los siguientes ítems: (1) Afronto la audición con atención; (2) Tengo conciencia de mi mism@ como escuchante; (3) Mi mente está distraída mientras oigo esta música; (4) Intento dirigir mi atención auditiva hacia algunos elementos de la música; (5) Trato de identificar algunos parámetros en lo que voy a escuchar; (6) Tengo unas pautas previas de escucha; (7) Trato de ordenar lo que escucho en base a unas categorías; (8) Trato de identificar cuáles son los parámetros más destacados de la audición; (9) Percibo bien los distintos parámetros de la audición: intensidad, velocidad, altura, timbres de los instrumentos...; (10) La música provoca en mí sensaciones físicas.

En segundo lugar, el nivel afectivo recoge afirmaciones relacionadas con la cualidad expresiva de la música, susceptible de provocar en quienes escuchan reacciones vinculadas al ámbito psicológico y sensitivo. Para ello se busca que las respuestas se ajusten a reflexiones acerca del aspecto gozoso de la escucha y también de los efectos que la música puede provocar, tales como agrado, impresiones más o menos fuertes, relajación, determinados sentimientos o confort. Además, interesa conocer si los escuchantes conectan fácilmente con la música e incluso si se sienten conmovidos o transportados por ella o si, aun no siendo así, reconocen su poder evocador de sentimientos. El alfa de Cronbach aumenta a 0.929 y las afirmaciones que componen la sub-escala afectiva son las siguientes: (1) Me siento a gusto como escuchante; (2) Lo que escucho me agrada; (3) Lo que escucho me impresiona; (4) Esta música me relaja; (5) Lo que escucho me conmueve; (6) La música provoca en mí algunos sentimientos; (7) Siento que la música me transporta; (8) Me siento en comunión con la música; (9) Esta música me hace sentir bien; (10) Esta música expresa sentimientos. 
Por último, el nivel analítico contiene declaraciones relacionadas con la captación de la organización del discurso musical y la atribución de un significado a lo escuchado. En ellas se recogen una serie de operaciones cognitivas tales como la atención, la identificación, el reconocimiento, la relación entre elementos, la búsqueda de un sentido o la comprensión de la funcionalidad de los parámetros y de su articulación con fines sintácticos y semánticos, que permiten inferir si los escuchantes, a partir de unos puntos de referencia facilitados con anterioridad, han adquirido unas competencias de comprensión de la música. El alfa de Cronbach continúa aumentando hasta 0.941 y el grupo de ítems asociado es el siguiente: (1) Trato de entender la estructura de esta pieza; (2) Presto atención al estilo de la música; (3) Intento identificar en qué época se escribió esta música; (4) Trato de reconocer a qué género pertenece esta pieza; (5) Trato de identificar a qué compositor pertenece esta música; (6) Trato de imaginar con qué finalidad se escribió esta pieza; (7) Trato de relacionar unos elementos con otros dentro de la audición para intentar comprender lo que escucho; (8) Esta música me hace pensar; (9) Esta música tiene un sentido / significado para mí; (10) Comprendo la función de algunos parámetros musicales (ritmo, melodía, altura, intensidad, timbres instrumentales...) en esta pieza.

Nunnally (1987) afirma que el alfa de Cronbach debe ser superior a 0,7 , por lo que, dado los resultados obtenidos, se considera que tanto la escala en su conjunto $(0,951)$ como las tres subescalas (sensorial 0,82; afectivo 0,929 y analítico 0,941) son fiables.

Además de la escala, el cuestionario recogía también si el encuestado tenía o no estudios musicales, así como su fecha de nacimiento, que servía de código de identificación anónimo del individuo.

\subsection{Procedimiento}

El cuestionario fue administrado en el aula en formato papel a la totalidad de los dos grupos de personas mayores que participaban en los dos cursos de OFM ofertados en la Universidad de Deusto durante el segundo semestre del curso 2015-2016. En ambos casos, previamente a la recogida de los datos, se informó a los alumnos y alumnas del carácter anónimo, confidencial y voluntario de su participación. Con el objetivo de medir el impacto de la intervención a través del OFM, el cuestionario se administró el primer y el último día de clase tras la audición de un fragmento musical correspondiente al Trío del Menuetto del Cuarteto de cuerda no 2 en La Mayor de Juan Crisóstomo Arriaga. Tal y como se desprende de lo anterior, tanto para la primera como para la última medición, la pieza musical utilizada para la audición fue exactamente la misma.

Para poder emparejar los cuestionarios anteriores y posteriores a la intervención en OFM, se habilitó un campo específico que recogía la fecha de nacimiento. Dicha fecha sirvió de código de enlace, de manera que únicamente se consideraron válidos aquellos cuestionarios para cuyo código específico existiera tanto un cuestionario inicial como otro final. Un total de 16 cuestionarios correspondían a alumnos que asistieron a la primera clase, pero no a la última o viceversa y, por tanto, como no cumplían el criterio establecido, ni siquiera se registraron en la base de datos.

Adicionalmente, se revisó la calidad de las respuestas y 11 parejas de cuestionarios se consideraron no válidas por contener ítems en blanco. Finalmente, fueron 37 las parejas de cuestionarios seleccionadas para el análisis, dentro de las cuales el 35\% de las personas encuestadas tenía algún tipo de estudios musicales (13 respuestas) y el $65 \%$ no (24 respuestas).

\subsection{Análisis de datos}

Una vez emparejados los cuestionarios en papel a través de la fecha de nacimiento, se llevó a cabo una digitalización de las respuestas a través de un formulario específico creado al efecto en la aplicación Google Forms. Dicha aplicación permite un fácil registro de los datos, creando al mismo tiempo una base de datos en Excel que posteriormente puede ser tratada o importada en sistemas estadísticos específicos. El formulario creado en Google Forms recogía de forma conjunta las respuestas de las dos mediciones realizadas. Así, las variables correspondientes a las escalas de los niveles de escucha se duplicaron para recoger la medición pre-OFM y post- OFM. Tras la digitalización de respuestas, la base de datos Excel se importó a la aplicación estadística SPSS (versión 21), creándose así la base de datos definitiva para el análisis de datos.

Para cada uno de los individuos se obtuvo el promedio de cada sub-escala (nivel sensorial, afectivo y analítico) en su aplicación pre y post intervención OFM. El análisis de cada nivel de escucha se realizó por separado, comparando las medias obtenidas para cada nivel antes y después de la intervención. Para entender la significatividad de la diferencia se aplicó el estadístico de la $t$-student para muestras relacionadas. La prueba se realizó tanto para el conjunto de la muestra $(n=37)$, como para los subconjuntos de individuos con estudios musicales previos $(n=13)$ y sin ellos $(n=24)$. 


\section{Resultados}

El análisis de resultados que se presenta a continuación se divide en tres subapartados, uno para cada nivel de escucha estudiado: sensorial, afectivo y analítico. En cada caso se muestra una tabla resumen con los datos del p-valor (campo denominado " $t$-Student"), que indican si los resultados del test de la $t$-student son significativos o no. Los resultados significativos se han señalado en la tabla con un asterisco $\left({ }^{*}\right)$ y se corresponden con valores del p-valor igual o menor a 0.05. Adicionalmente se presentan también la media y desviación estándar de la escala en la aplicación del cuestionario antes de la intervención (pre-OFM), así como la media y desviación estándar de la escala en la aplicación del cuestionario después de la intervención (post-OFM).

Cada una de las tablas presenta los resultados para la totalidad de la muestra $(n=37)$ y también para el subconjunto de encuestados que no contaba con estudios musicales $(n=24)$ y el que sí contaba con ellos $(n=13)$.

\subsection{Nivel sensorial}

En el caso del nivel sensorial, a pesar de que las medias de la escala son superiores tras la intervención en OFM, las diferencias no son estadísticamente significativas, tal y como se puede observar en los resultados del test de la t-Student.

\begin{tabular}{|l|c|c|c|c|c|c|}
\hline \multicolumn{7}{|c|}{ Tabla 1: Prueba t para muestras relacionadas - Nivel sensorial } \\
\hline Colectivos & \multirow{2}{*}{$\mathrm{N}$} & \multicolumn{2}{|c|}{ PRE-OFM } & \multicolumn{2}{c|}{ POST-OFM } & \multirow{2}{*}{$t$-Student } \\
\cline { 3 - 6 } & & Media & $\begin{array}{c}\text { Desviación } \\
\text { estándar }\end{array}$ & Media & $\begin{array}{c}\text { Desviación } \\
\text { estándar }\end{array}$ & \\
\hline Sin estudios musicales & 24 & 4,7163 &, 91965 & 4,8528 &, 85879 & .640 \\
\hline Con estudios musicales & 13 & 4,9615 & 1,12734 & 5,1771 &, 94660 & .330 \\
\hline Total & 37 & 4,8025 &, 98897 & 4,9667 &, 89136 & .415 \\
\hline
\end{tabular}

Los resultados obtenidos señalan que la intervención a través del OFM no afecta al nivel sensorial.

\subsection{Nivel afectivo}

En el nivel afectivo ocurre lo mismo que en el nivel sensorial, si bien las diferencias entre las medias pre y post intervención en OFM son todavía menores. De hecho, los valores son muy similares e, incluso, se puede observar que en el caso del colectivo con estudios musicales la media post es inferior a la media pre. De nuevo, el test de la $t$-Student corrobora que las diferencias entre las medias no son significativas.

\begin{tabular}{|c|c|c|c|c|c|c|}
\hline \multirow[b]{2}{*}{ Colectivos } & \multirow[b]{2}{*}{$\mathrm{N}$} & \multicolumn{2}{|c|}{ PRE-OFM } & \multicolumn{2}{|c|}{ POST-OFM } & \multirow[b]{2}{*}{$t$-Student } \\
\hline & & Media & $\begin{array}{l}\text { Desviación } \\
\text { estándar }\end{array}$ & Media & $\begin{array}{l}\text { Desviación } \\
\text { estándar }\end{array}$ & \\
\hline Sin estudios musicales & 24 & 5,7287 & 1,00519 & 5,8323 & 1,05316 & .649 \\
\hline Con estudios musicales & 13 & 5,8615 &, 90142 & 5,7342 & ,90779 & .620 \\
\hline Total & 37 & 5,7754 & ,95944 & 5,7978 & ,99276 & .895 \\
\hline
\end{tabular}

El análisis de resultados indica que la intervención a través del OFM no afecta al nivel afectivo. 


\subsection{Nivel analítico}

El nivel analítico invierte la tendencia encontrada hasta el momento, dado que en este caso las diferencias entre las medias pre y post son lo suficientemente amplias como para que el test de la $t$-Student establezca que los resultados son estadísticamente significativos. En concreto, los resultados son significativos para el conjunto del colectivo y para el subconjunto de personas sin estudios musicales. Sin embargo, los resultados no son significativos para el segmento que cuenta con estudios musicales.

\begin{tabular}{|c|c|c|c|c|c|c|}
\hline \multirow[b]{2}{*}{ Colectivos } & \multirow[b]{2}{*}{$N$} & \multicolumn{2}{|c|}{ PRE-OFM } & \multicolumn{2}{|c|}{ POST-OFM } & \multirow[b]{2}{*}{ t-Student } \\
\hline & & Media & $\begin{array}{l}\text { Desviación } \\
\text { estándar }\end{array}$ & Media & $\begin{array}{l}\text { Desviación } \\
\text { estándar }\end{array}$ & \\
\hline Sin estudios musicales & 24 & 3,7856 & 1,36425 & 4,4583 & ,87488 & $.005^{*}$ \\
\hline Con estudios musicales & 13 & 4,7806 & 1,02518 & 5,0291 & 1,17254 & .255 \\
\hline Total & 37 & 4,1352 & 1,33089 & 4,6589 & 1,01173 & $.003^{*}$ \\
\hline
\end{tabular}

Los resultados obtenidos señalan que la intervención a través del OFM sí afecta al nivel analítico, especialmente en el caso de aquellas personas $\sin$ estudios musicales.

\section{Discusión y conclusiones}

Tras la revisión de literatura y el análisis de resultados realizados, destaca, en primer lugar, la evidencia encontrada de la naturaleza subjetiva de la respuesta al estímulo musical (Small, 2006). Las contestaciones a los ítems planteados y el grado de acuerdo o desacuerdo con su contenido, que ofrecen los sujetos de la muestra y que quedan reflejados en las escalas Likert, recogen la riqueza de la variedad. Esto obedece, sin duda, a los múltiples factores individuales que emanan de cualquier grupo humano (MacDonald, Hargreaves \& Miell, 2012; Sloboda, 2012; Szendy, 2015) y, más aún, de los grupos heterogéneos de personas que toman contacto con este tipo de programas de ocio formativo (Cámara, Cañada, Albaina \& Larrinaga, 2012). Hay singularidades, consustanciales a la idiosincrasia de cada persona, que no son susceptibles de manipulación por parte del educador - conductor de la escucha (Ball, 2010; Blacking, 2006; Dahlhaus \& Eggebrecht, 2012; Drösser, 2012; Levitin, 2011; Sacks, 2015). También cabe destacar la existencia otro tipo de diferencias como las referidas a habilidades auditivas, estudios musicales, actitud hacia la música y biografía musical que, si bien no son inamovibles y pueden ser matizadas a través de la audición y la educación musical, marcan unos colectivos caracterizados por la diversidad (Bonds, 2014; Horowitz, 2012; Swanwick, 2006). Esto, indudablemente y en beneficio de la eficiencia educativa, condiciona los planteamientos didácticos a la hora de diseñar los programas de OFM (Albaina, 2015; Delalande, 2013; Schafer, 2013).

En segundo lugar, y partiendo del planteamiento de que el hecho musical es captado en tres niveles de escucha complementarios, la presente investigación concluye que el único nivel de escucha afectado de manera significativa tras la intervención de OFM estudiada es el analítico y, afinando más, el impacto solo es destacable para los individuos de la muestra sin estudios musicales. Aparentemente, el ámbito de actuación del OFM es tangencial a las competencias que implica el nivel sensorial de escucha. En consecuencia, aun considerando la importancia de los procesos perceptivos en la base del complejo fenómeno de la audición (Ball, 2010; Bonds, 2014; Boulez, Changeux \& Manoury, 2016; Levitin, 2011; Sacks, 2015; Sloboda, 2012; Small, 2006; Szendy, 2015), se constata que la incidencia del programa de OFM analizado, no es destacable en este primer nivel de escucha. El nivel afectivo muestra resultados similares, ya que la esencia formativa y de ocio del programa analizado no parece afectar a la solidez de las experiencias subjetivas que las personas experimentan con la música en el plano de las emociones. Esta identificación con la cualidad expresiva de la música, recogida de la literatura de referencia, apenas cambia de signo en ninguno de los dos colectivos en que se ha dividido la muestra, lo que apunta que este nivel 
de escucha se mantiene en el plano de la inconsciencia (Swanwick, 2006) y de la esencia misma de cada individuo (Benenzon, 2011), que es afectado por la música en su particular implicación emocional con ella (Gabrielsson, 2011).

Por último, en lo referente al nivel analítico, se observa que las pautas de escucha que se proporcionan a los oyentes en los programas de OFM, tienen una incidencia que queda reflejada en las diferencias entre las medidas previas a la intervención y las posteriores (Albaina, 2015; Boulez, Changeux \& Manoury, 2016). De los resultados obtenidos cabe inferir que el plano cognitivo se ve estimulado y que la índole didáctica del OFM es eficaz fundamentalmente en aquellos no alfabetizados musicalmente (Ball, 2010; Levitin, 2011; Sloboda, 2012). De esta manera, se confirma la bondad y la utilidad de los programas de OFM en el enriquecimiento del panorama educativo musical -dentro del contexto no reglado de la educación- y en los planteamientos tan en boga y valiosos del aprendizaje a lo largo de la vida (Vargas, 2017). Los puntos de referencia que se adquieren en el marco del OFM, permiten analizar -en mayor o menor grado de profundidad-el discurso musical y ayudan a las personas a comprenderlo al atribuirle una significación (Ball 2010; Sacks, 2015; Sloboda, 2012; Swanwick, 2006). Por tanto, si bien los tres niveles de escucha están enlazados y son indisociables, es en este nivel auditivo donde se evidencia el impacto de estos programas, que transforman al oyente en escuchante (Albaina, 2015; Boulez, Changeux \& Manoury, 2016; Copland, 2008; Dahlhaus \& Eggebrecht, 2012; Delalande, 2013).
Como limitaciones a este trabajo cabe señalar el tamaño de la muestra y la longitud del cuestionario. La muestra, a pesar de abarcar dos grupos diferentes con un total de 64 individuos se ha visto reducida a 37 respuestas válidas. La dificultad de recoger cuestionarios el primer y el último día de clase, unido a la longitud y dificultad del cuestionario, ha contribuido a elevar el número de registros perdidos. Por ello, como futuras líneas de intervención se propone utilizar los datos existentes para simplificar la escala a través de análisis factoriales sin perjudicar el nivel de fiabilidad. La necesidad de tener que contestar a 10 ítems para cada nivel de escucha ha generado dificultades en los encuestados, ya que a veces les costaba diferenciar un ítem de otro. La simplificación de la escala tendría probablemente un impacto positivo en la administración del cuestionario y recogida de respuestas. Una vez realizado lo anterior, sería recomendable identificar diferentes programas de OFM más allá del contexto concreto aquí estudiado, para llegar a un colectivo más amplio y poder contrastar las conclusiones que apunta la presente investigación. Así mismo, otra posible línea de investigación sería la profundización en el impacto de los programas de OFM en el nivel afectivo y su relación con el nivel analítico. Varios autores (Drösser, 2012; MacDonald, Hargreaves \& Miell, 2012; Sloboda, 2012) defienden que las expectativas del escuchante y los factores contextuales, pueden favorecer las experiencias emocionales respecto a la música. A pesar de que esta tesis no ha podido ser confirmada a través de este estudio, resultaría de gran interés profundizar en esta área en investigaciones futuras.

\section{Referencias bibliográficas}

Abellán, A., \& Ayala, A. (2012). Un perfil de las personas mayores en España, 2012. Indicadores estadísticos básicos. Informes Portal Mayores, 131 Obtenido de http://envejecimiento.csic.es/documentos/documentos/ pm-indicadoresbasicos12.pdf (última consulta: 17 de marzo de 2013).

Albaina, M. (2015). Ocio Formativo Musical Análisis de iniciativas en los Programas Universitarios para Mayores y en las Orquestas Sinfónicas de España. Tesis doctoral no publicada. Bilbao: Universidad de Deusto.

Albaina, M. \& Cámara, A. (2010). Training New Listeners for Music. Obtenido de http://depts.washington.edu/icmpc11/ ICMPC11_ABSTRACTS.pdf (última consulta: 13 de julio de 2017).

Alvin, J. (1997). Musicoterapia. Barcelona: Paidós.

Ball, Ph. (2010). El instinto musical: escuchar, pensar y vivir la música. Madrid: Turner.

Benenzon, R.O. (2011). Musicoterapia: De la teoría a la práctica. Barcelona: Paidós lbérica.

Blacking, J. (2006). ¿Hay música en el hombre? Madrid: Alianza.

Blasco, F. (1996). Evaluación de los efectos psicológicos de la música a través de un diferencial semántico. Revista Brasileira de Musicoterapia Año I (2), 5-23.

Boerner, S., \& Jobst, J. (2013). Enjoying Theater: The Role of Visitor's Response to the Performance. Psychology of Aesthetics, Creativity, and the Arts, 7(4), 391- 408.

Bonds, M.E. (2014). La música como pensamiento. Barcelona: Acantilado.

Boulez, P, Changeux J.P., \& Manoury, Ph. (2016). Las neuronas encantadas. El cerebro y la música. Barcelona: Gedisa. 
Cámara, A., Cañada, M., Albaina, M., \& Larrinaga, I. (2012). Programas educativos musicales en la CAPV: Miradas desde la diversidad de públicos. Obtenido de http://www.eusko-ikaskuntza.org/es/publicaciones/acercar-la-musica-a-traves-de-programas-educativos-en-la-capv-miradas-desde-la-diversidad-de-publicos/ar-22320/ (última consulta: 13 de julio de 2017).

Chin, T. Ch., \& Rickard, N. S. (2012). The Music USE (MUSE) Questionnaire: An Instrument to Measure Engagement in Music. Music Perception, 29(4), 429-446. doi: 10.1525/mp.2012.29.4.429.

Copland, A. (2008). Cómo escuchar la música. Madrid: Fondo de Cultura Económica.

Cuenca, M. (2014). Ocio valioso. Bilbao: Universidad de Deusto.

Cuenca-Amigo, M., \& San Salvador del Valle, Roberto. (2016). La importancia del ocio como base para un envejecimiento activo y satisfactorio. Revista de Psicología del Deporte, 25 (Suppl 2), 79-84.

Dahlhaus, C., \& Eggebrecht, H.H. (2012). ¿Qué es la música? Barcelona: Acantilado.

Delalande, F. (2013). Las conductas musicales. Santander: Ediciones Universidad de Cantabria.

Drösser. Ch. (2012). La seducción de la música. Los secretos de nuestro instinto musical. Barcelona: Ariel.

Gabrielsson, A. (2002). Old People's Remembrance of Strong Experiences Related to Music. Psychomusicology 18, 103-122.

Gabrielsson, A. (2011). Strong experiences with music. Nueva York: Oxford University Press.

González, J. A., \& Pazmiño, M. (2015). Cálculo e interpretación del Alfa de Cronbach para el caso de validación de la consistencia interna de un cuestionario, con dos posibles escalas tipo Likert. Revista Publicando, 2(7), 62-77.

Horowitz, S. S. (2012). The universal sense: how hearing shapes the mind. New York: Bloomsbury.

Law, L. N. C., \& Zentner, M. (2012). Assessing musical abilities objectively: construction and validation of the profile of music perception skills. PLoS ONE, 7(12): e52508. https://doi.org/10.1371/journal.pone.0052508.

Levitin, D. (2011). Tu cerebro y la música. Barcelona: RBA.

MacDonald, R., Hargreaves, D.J., \& Miell, D. (2012). Musical identities mediate musical development. In McPherson, G. \& Welch, G. (Eds.) Oxford Handbook of Music Education. vol. 1, Oxford: Oxford University Press. doi: 10.1093/oxfordhb/9780199730810.013.0008.

Malhotra, N. K. (2004). Investigación de mercados: un enfoque aplicado. (4a ed.). México: Pearson Educación.

Meyer, L.B. (2009). Emoción y significado en la música. Madrid: Alianza.

Nunnally, J. C. (1987). Teoría Psicométrica. México: Trillas.

Rosen CH. (2012). Música y sentimiento. Madrid: Alianza.

Rössel, J. (2011). Cultural capital and the variety of Modes of cultural consumption in the opera audience. The Sociological Quarterly 52, 83-103.

Sacks, O. (2015). Musicofilia: Relatos de la música y el cerebro. Barcelona: Anagrama.

Schafer, R.M. (2013). El paisaje sonoro y la afinación del mundo. Barcelona: Intermedio.

Sloboda, J. (2012). La mente musical. La psicología cognitiva de la música. Madrid: Machado Nuevo Aprendizaje.

Small, Ch. (2006). Música, sociedad, educación. Madrid: Alianza.

Swanwick, K. (2006). Música, pensamiento y educación. Madrid: Morata.

Szendy, P. (2015). En lo profundo de un oído. Santiago de Chile: Metales Pesados.

Thompson, S. (2007). Determinants of listeners' enjoyment of a performance. Psychology of Music 35(1), 20-36.

Vargas, C. (2017). El aprendizaje a lo largo de toda la vida desde una perspectiva de justicia social(21). París: UNESCO. Obtenido de https://es.unesco.org/node/262744 (última consulta: 9 de septiembre de 2017).

Willems, E. (2001). El oído musical. Barcelona: Paidós.

\section{Nota}

1 El término nivel, tal como se utiliza aquí, es sinónimo de rango o categoría y lleva asociada la idea de gradación o profundidad en la escucha, en función del nivel en que el oyente se sitúe. En este sentido, el orden de gradación sería el siguiente: (1) sensorial; (2) afectivo y (3) analítico. 


\section{CÓMO CITAR ESTE ARTÍCULO}

Cuenca-Amigo, M., \& Albaina, M. (2018). Impacto del ocio formativo musical en los niveles de escucha. Resultados de una intervención con personas mayores. Pedagogía Social. Revista Interuniversitaria, 32 127-137. DOI:10.7179/PSRI_2018.32.09

\section{DIRECCIÓN COMPLETA DE LOS AUTORES}

Macarena Cuenca-Amigo. Universidad de Deusto, Deusto Business School. Hermanos Aguirre 2, 48014 Bilbao, España. Tel. 944139000. E-mail. macarena.cuenca@deusto.es

Mercedes Albaina. Musikene. Europa Plaza, 2, 20018 Donostia, España. Tel. 943023750 . E-mail. malbaina@musikene.net

\section{PERFIL ACADÉMICO}

Macarena Cuenca-Amigo. Doctora en Ocio y Desarrollo Humano. Profesora en Deusto Business School y miembro del Equipo de Investigación del Instituto de Estudios de Ocio de la Universidad de Deusto. Su principal línea de investigación es el desarrollo de audiencias culturales, tema sobre el cual desarrolló su tesis doctoral, imparte docencia de postgrado en diversas universidades y participa en varios proyectos financiados por la Comisión Europea. Ha publicado en revistas, tales como: Academia Revista Latinoamericana de Administración, Annals of Leisure Research, Arbor, Cuadernos de Gestión, International Review of Social Research, Revista de Psicología del Deporte.

Mercedes Albaina. Titulada Superior en Música, Doctora en Ocio y Desarrollo Humano y Licenciada en Psicología. Profesora titular de Educación Auditiva en Musikene y profesora visitante en el Instituto de Ocio de la UD. Contribuye a la divulgación de la Música a través de conferencias, escritos y proyectos didácticos. Ponente en diversos Congresos y Jornadas nacionales e internacionales de Investigación y Educación Musical. Colabora con entidades públicas y privadas en formación del profesorado de música. Su tesis doctoral recibió el premio Orfeón Donostiarra-UPV/ EHU. Galardonada con el premio AixeGetxo a la cultura, en la modalidad de Música clásica y contemporánea y con el Premio 'Adoración Holgado Sánchez' de investigación de la Asociación Estatal de Programas Universitarios para Personas Mayores. 
